\title{
Scalable Data Processing for Prediction, Batch Computation and Analysis and Response Times using Google BigQuery
}

\author{
Gitanjali Sinha $^{1}$,Dr. Asha Ambhaikar ${ }^{2}$ \\ ${ }^{1}$ Ph.D Scholar, Department of Computer Science, kalinga university Raipur, (Chhattisgarh), INDIA \\ (Email id:.sinha.gitu@gmail.com) \\ ${ }^{2}$ Professor\& HOD(CSE) \& Dean Student Welfare, Department of Computer Science, kalinga \\ university,Raipur, (Chhattisgarh), INDIA \\ (Email id:dr.asha.ambhaikar@gmail.com)
}

\begin{abstract}
:
Computing a complex dataset analysis is tedious task because distributed resource management is difficult task. Google disperses the registering utilized by BigQuery across process assets powerfully which implies that we don't need to oversee figure asset, for example, bunches, register motor, stockpiling structure. Fighting commitments customarily require custom estimating (and esteeming) of unequivocal procedure gatherings, and this can change after some time which can be trying. Since Google logically assigns resources, costs are dynamic too. Google offers both a compensation all the more just as costs emerge elective where you pay for the data brought into BigQuery and subsequently per question costs. Since BigQuery is a totally managed organization, the backend game plan and tuning is managed by Google. This is much more direct than battling plans that anticipate that you should pick a number and sort of gatherings to make and to administer after some time. BigQuery consequently recreates information between zones to empower high accessibility. It additionally naturally load adjusts to give
\end{abstract}

ideal execution and to limit the effect of any equipment disappointments. So getting benefits of BigQuery we did complex data analysis in huge amount of data set within a friction of second. Our result is showing the capability of our research work in the field of scalable data processing.

keyword: BigQuery, ETL(Extraction, Transformation, load), Batch Processing, distributed processing etc.

Introduction :

Google Cloud comprises of a lot of physical resources, for example, PCs and hard plate drives, and virtual assets, for example, virtual machines (VMs), that are contained in Google's server farms the world over. Every server farm area is in a locale. Locales are accessible in Asia, Australia, Europe, North America, and South America. Every locale is an assortment of zones, which are detached from one another inside the area[1]. Each zone is recognized by a name that joins a letter identifier with the name of the area. 
BigQuery is Google's completely oversaw, petabyte scale, minimal effort examination information distribution center. BigQuery is NoOps - there is no foundation to oversee and you needn't bother with a database manager - so you can concentrate on breaking down information to discover significant bits of knowledge, utilize recognizable SQL, and exploit our pay-more only as costs arise model. Serverless, exceptionally adaptable, and financially savvy multi-cloud information distribution center intended for business readiness[2]. Some of the main features include-

- Analysing petabytes of information utilizing ANSI SQL at bursting quick speeds, with zero operational overhead.

- Run investigation at scale with $27 \%-35 \%$ lower three-year TCO than cloud information distribution center other options.
- Democratize bits of knowledge with a trusted, and safer stage that scales with your necessities.

- Increase bits of knowledge from information across mists with an adaptable, multi-cloud examination arrangement.

\section{BigQuery Architecture}

BigQuery is based on head of Dremel innovation which has been underway inside in Google since 2006. Dremel is Google's intelligent impromptu inquiry framework for examination of read-just settled information. Unique Dremel papers were distributed in 2010 and at the hour of distribution Google was running numerous cases of Dremel going from tens to thousands of hubs [3].

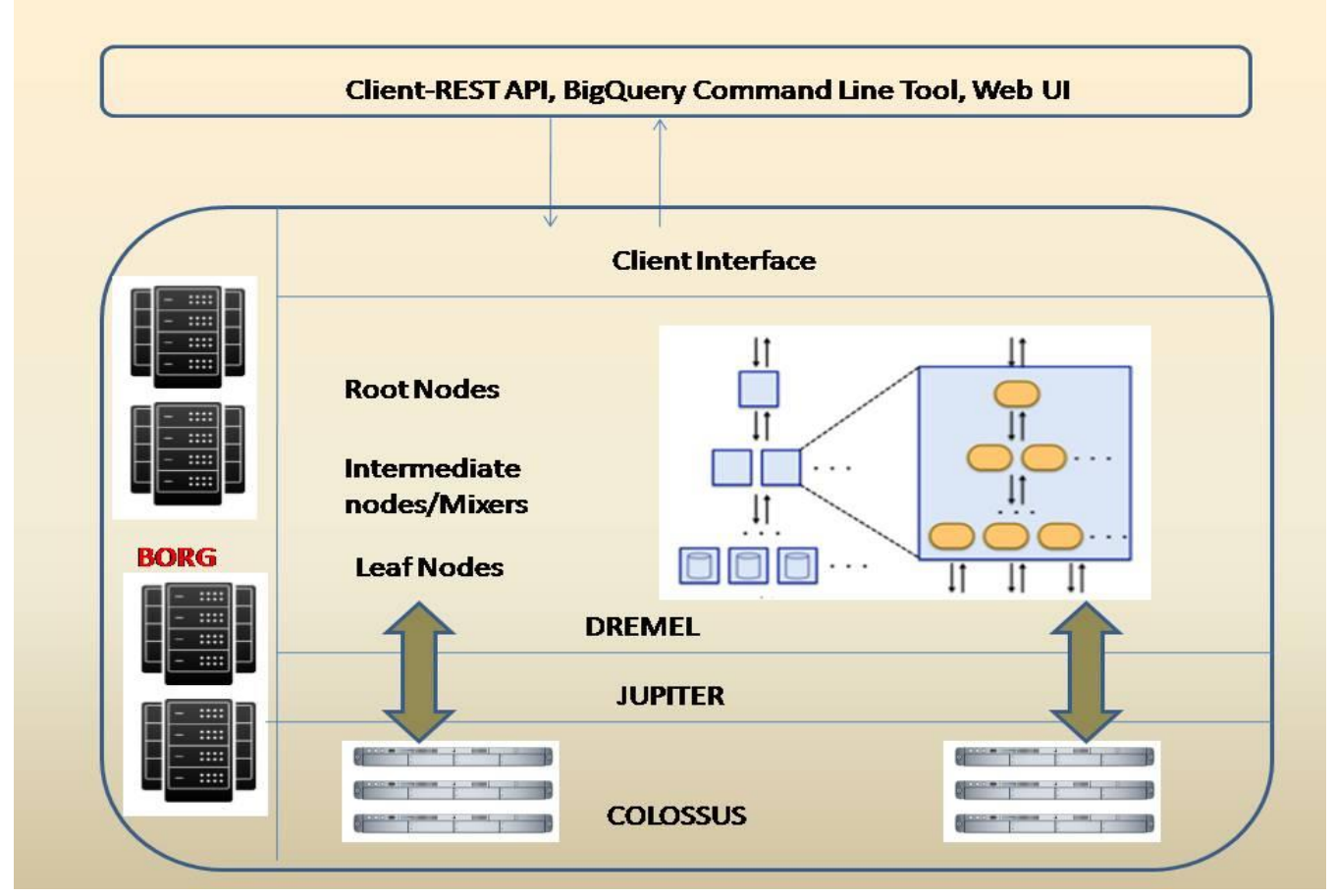

Figure 1 High level BigQuery Architecture Based on Dremel Technology. 
BigQuery and Dremel share the equivalent basic design. By fusing columnar capacity and tree design of Dremel, BigQuery offers uncommon execution. Be that as it may, BigQuery is considerably more than Dremel. Dremel is only an execution motor for the BigQuery. Indeed, BigQuery administration use Google's imaginative advancements like Borg, Colossus, Capacitor, and Jupiter. As showed underneath, a BigQuery customer (regularly BigQuery Web UI or bg order line device or REST APIs) cooperate with Dremel motor by means of a customer interface. Borg - Google's huge scope group the board framework - allots the register limit with respect to the Dremel occupations. Dremel occupations read information from
Google's Colossus document frameworks utilizing Jupiter arrange, perform different SQL activities and return results to the customer. Dremel actualizes a staggered serving tree to execute questions which are shrouded in more detail in following areas.

\section{Comparison Between BiqQuery and MapReduce}

BigQuery and MapReduce are in a general sense various advances and every has diverse use belongings. The accompanying board thinks about the two advances and and finding aout suitable place to apply.

Table 1 Comparison Between BiqQuery and MapReduce

\begin{tabular}{|l|l|l|}
\hline Terms & MapReduce & BigQuery \\
\hline What's going on here? & $\begin{array}{l}\text { Programming model for handling } \\
\text { enormous datasets }\end{array}$ & $\begin{array}{l}\text { Inquiry administration for } \\
\text { enormous datasets }\end{array}$ \\
\hline Normal use cases & $\begin{array}{l}\text { what's more, investigating Clump } \\
\text { preparing of enormous dataset for } \\
\text { tedious information transformation or } \\
\text { total }\end{array}$ & $\begin{array}{l}\text { Specially anpointed } \\
\text { experimentation } \\
\text { question of enormous dataset for } \\
\text { fast examination }\end{array}$ \\
\hline OLAP/BI use case & No & Yes \\
\hline Data Mining use case & Yes & Partially \\
\hline Fast Response Time & Yes & No \\
\hline $\begin{array}{l}\text { Easy to use for non- } \\
\text { programmers }\end{array}$ & NO & No \\
\hline $\begin{array}{l}\text { Programming complex } \\
\text { data processing logic }\end{array}$ & Yes & Partially \\
\hline $\begin{array}{l}\text { Processing unstructured } \\
\text { data }\end{array}$ & Yes & No \\
\hline Handling large results & Yes & \\
\hline
\end{tabular}




\begin{tabular}{|l|l|l|}
\hline Updating existing data & Yes & No \\
\hline
\end{tabular}

\section{literature Survey :}

Non-social database registering in Clouds is a new model for up and coming age investigation improvement, empowering unstructured information association, sharing, and investigation of huge volumes quickly developing assortment types of information utilizing Cloud processing innovations as a back end huge scale administration arranged computational framework office. Advances in data innovation and its broad development in various territories of business, building, clinical and logical examines are bringing about data and information blast. There are numerous strategies to deal with non-social database on cloud. This theory center around taking care of non-social database utilizing planning advance. This paper utilizes Google cloud administrations like huge inquiry [4]. Projected planning calculation was useful on intuitive and cluster question utilizing reserved information and without utilizing stored information. Proposed calculation lessens pausing time and along these lines improves the question execution time."

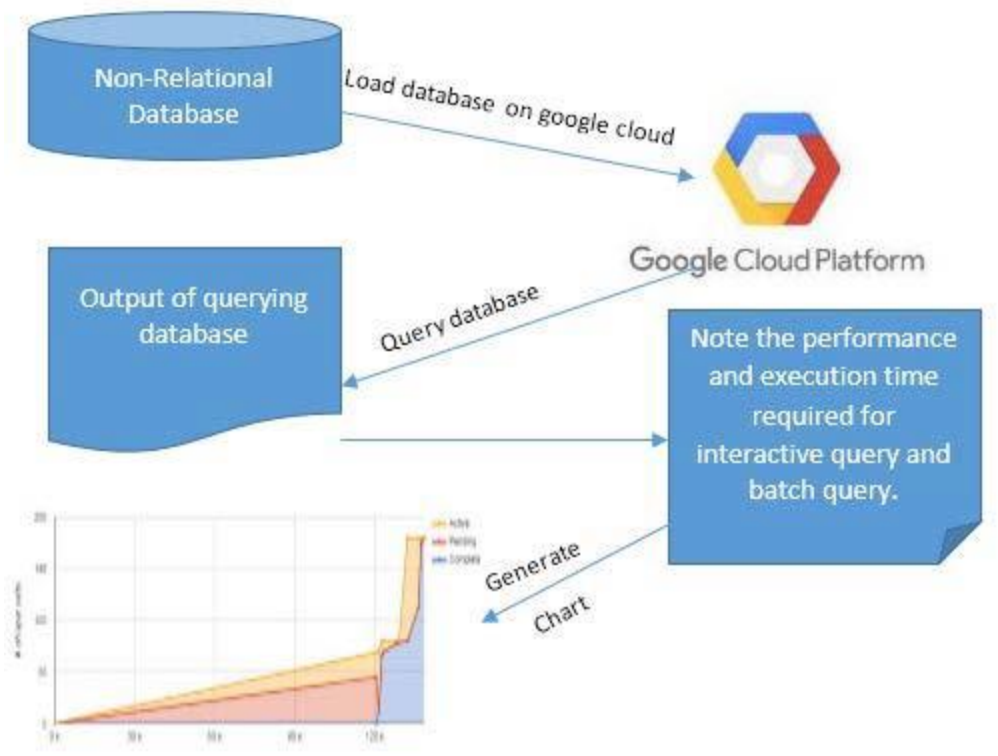

Figure 2 Proposed Architecture of Non - Sql Database analysis

More effect on immense database as stand out from little database [5]. Proposed research work perform marvelously in each situation imagine. The Proposed framework that can't execute proportionate solicitation in explicit conditions where step 2 information is relied on step 1 outcome. Here it is hard to start the equivalent step 2 where holding on for step 1.

So as to improve the accuracy and review of watchwords dynamic solicitation in social database, an amazing watchword demand figuring in social database subject to coalition 
attributes information drawing out and systematic recovery is planned, request composing PC programs is organized. To improve the request benefit, decay the time transparency of diagram investigate in demand procedure, the technique for way record is use to set up the catchphrases active solicitation replica of opposite interest on or after the watchword place and ahead solicitation from possible root community point, and concentrate the linked attributes of the watchwords in the social data[6].

Through the systematic recovery, the terms, focuses. Standard Linux improvement instruments are utilized for demand programming improvement and structure. The reenactment results show that the proposed philosophy can improve the precision and review of the dynamic catchphrase demand in the social database, and the arranging show of is better and the nonstop execution and exactness of the database question are refreshed [7].

This paper mulls over the watchword dynamic inquiry technique for in social database. Considering the colossal data examination strategy, an incredible catchphrase question computation in social database considering alliance traits data taking out and semantic recuperation is arranged, and the request writing

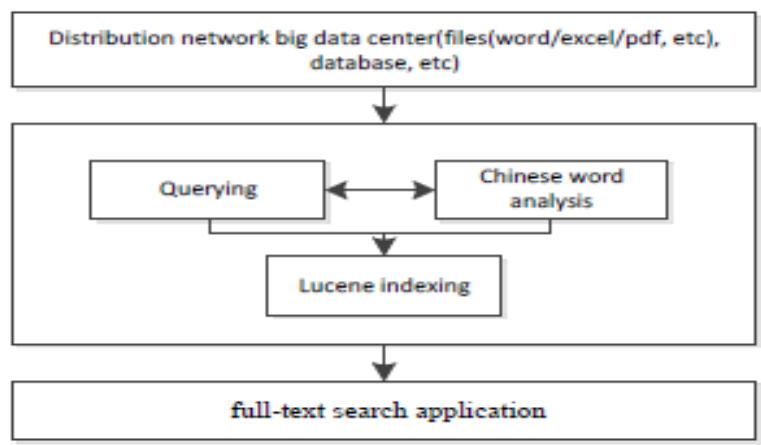

847 computer programs is arranged. Standard Linux movement instruments are utilized for question programming progress and structure[8].

Huge information is one of the most up to date and greatest innovative changes in the IT business. Information search, information affiliation and examination are the fundamental needs by large information utilities. There are a wide range of scholarly information in appropriation arrange which was not used along with investigated. Complete inquiry can join directly words and return group informational collection as indicated by the quest which gives the comfort to information the executives and choice help [9].

In this paper, a fulltext recovery framework dependent on Lucene design incorporated with Hadoop engineering for conveyance organize huge information focus is built up which could give scholarly and full-text inquiry over all dispersion organize the executives frameworks for example, Distribution the executives system(DMS), Intelligent framework for incorporated network activity (OS2), Customer administration framework and so on. 
Figure 3 Full text search article

The proposed application could help framework administrators to attach the data recovery and give the accommodation and effectiveness of information investigation by the large information innovation. Enormous information for the appropriation arrange gives both openings and difficulties. There is a colossal measure of abstract information in dispersion organize which has not been completely used and dissected [10]. The advantages of large information innovations could be bringing information see, changing reasoning strategies and apparatuses, expand the solicitation scene, giving better organization to the overall population by improving the value. Lucene et. al. has speedier speed and improved execution, It gets pace consolidate technique away. In Lucene structure, a couple of little records are developed, and by then the little records are joined into a gigantic document tree subsequent to everything looks at. Thus, it can work the full flash recuperation exclusive of spoiling the execution.

In this research paper, in light of Lucene, a complete recovery arrangement of conveyance framework enormous server farm is set up which could give abstract and full-text question over all the conveyance arrange the board frameworks. The full-text recovery framework could help framework administrators to secure the data recovery and give the accommodation of the enormous information innovation, for example, information demonstrating, examination and representation [11].

\section{Methodology :}

\section{Step - 1 Uploading dataset to Google Cloud Engine}

You can stack information in any arrangement for example JSON or may be CSV file. Precisely when you load information from Cloud Storage into a BigQuery table, the dataset that contains the table must be in the equivalent typical or multi-territorial zone as the Cloud Storage holder.

\section{Use web UI BigQuery.}

2. Cost on a dataset, click the down stun picture, and click create new table. The framework for stacking data is like the method for making an empty table.

3. On the Create Table page, in the Source Data piece: For File arrangement, select Commaisolated qualities (CSV). Note that you can bar distinctive URIs. 


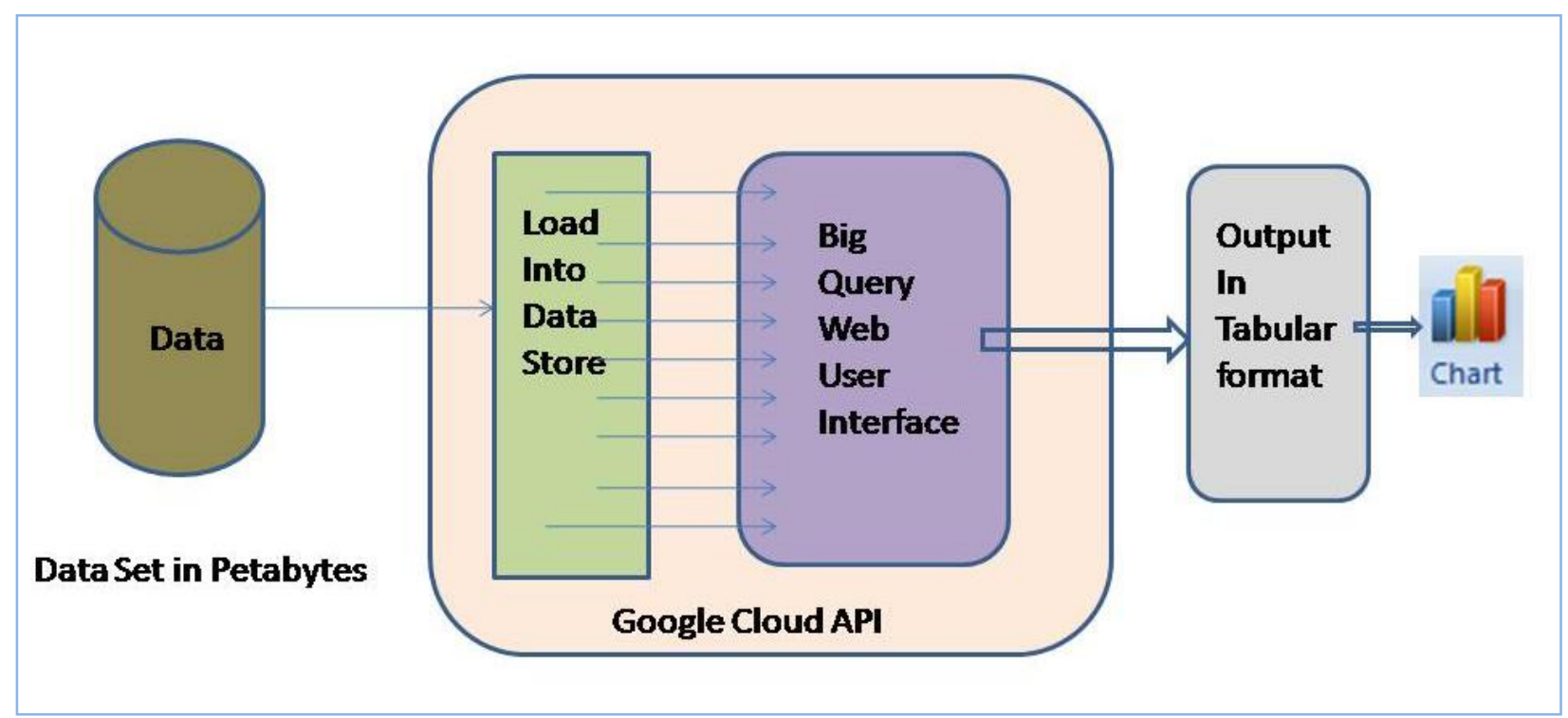

Figure 4 Methodology of Research work

4. In this Schema, go into the structure explanation. Using CSV records, we can check the recognize decision to connect with advancement auto-affirmation.

\section{Stage 2: Querying the Cloud database}

1. Utilizing Bigquery WEB,find in fig. 5 on the left side Question key, Click on it and make the solicitation in the new zone and snap on Run Query.

2. Utilizing the bq Command-Line Tool. This domain contain general data on utilizing the bq demand line contraption.

4. Authorized in google account.

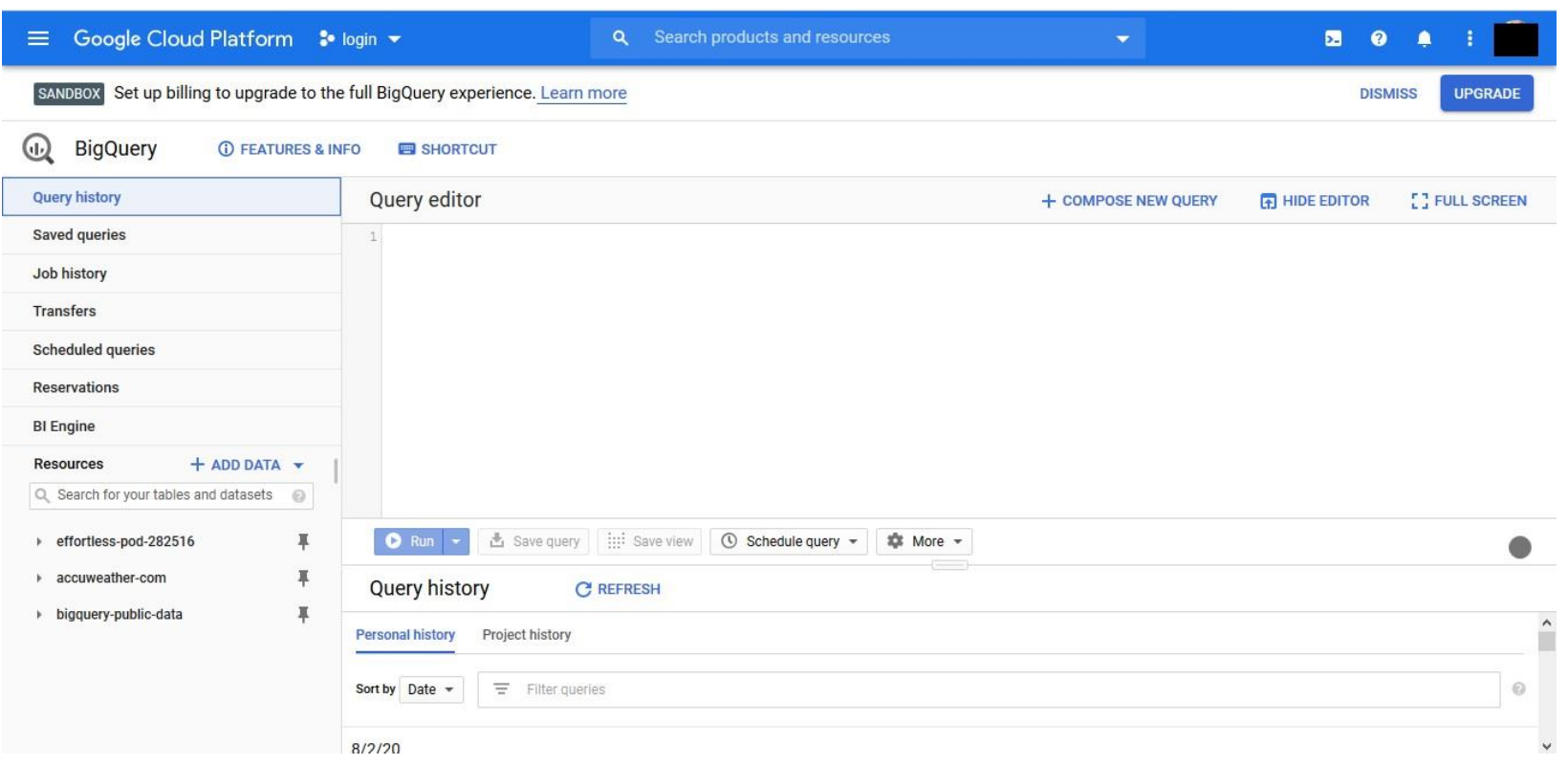




\section{Figure 5 BigQuery Web User interface}

\section{Stage 3: Observe the time required to execute}

For intuitive inquiry and group question "As appeared in Fig. 5, you can set the question execution boundaries like Interactive or bunch inquiry and you can likewise set that whether you need to utilize reserved information or you don't need to utilize stored information. Select the boundaries run the test information.

\section{Stage 4: Generate graph}

Produce the diagram in regards to which open information test took how much seconds to run the question. Likewise plot the diagram for intuitive and group question both. You can likewise recognize it by utilizing stored result and utilizing non stored result.

\section{Stage 5: Performance examination}

Run the inquiry for all example projects and note the results and contrast it and past outcome. Draw the end and plot the outline."

\section{Result :}

In this section we are finding a language which has the best community,based on the response time. For that we are having google public repository bigquery-publicdata.stackoverflow.

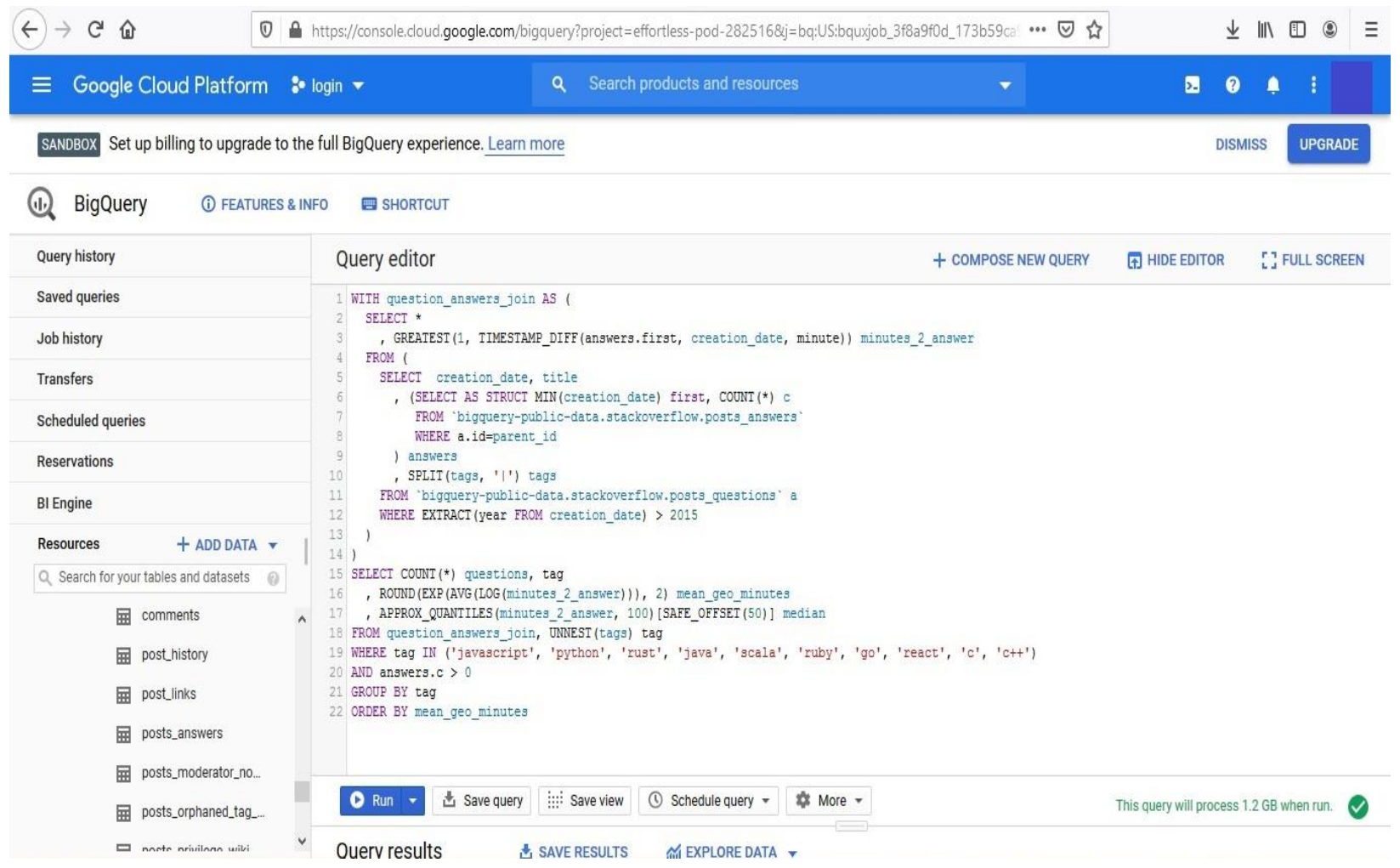

Figure 6 Fetching BigQuery in web UI 
Above figure is showing that how we are fetching the query into google cloud datastore.

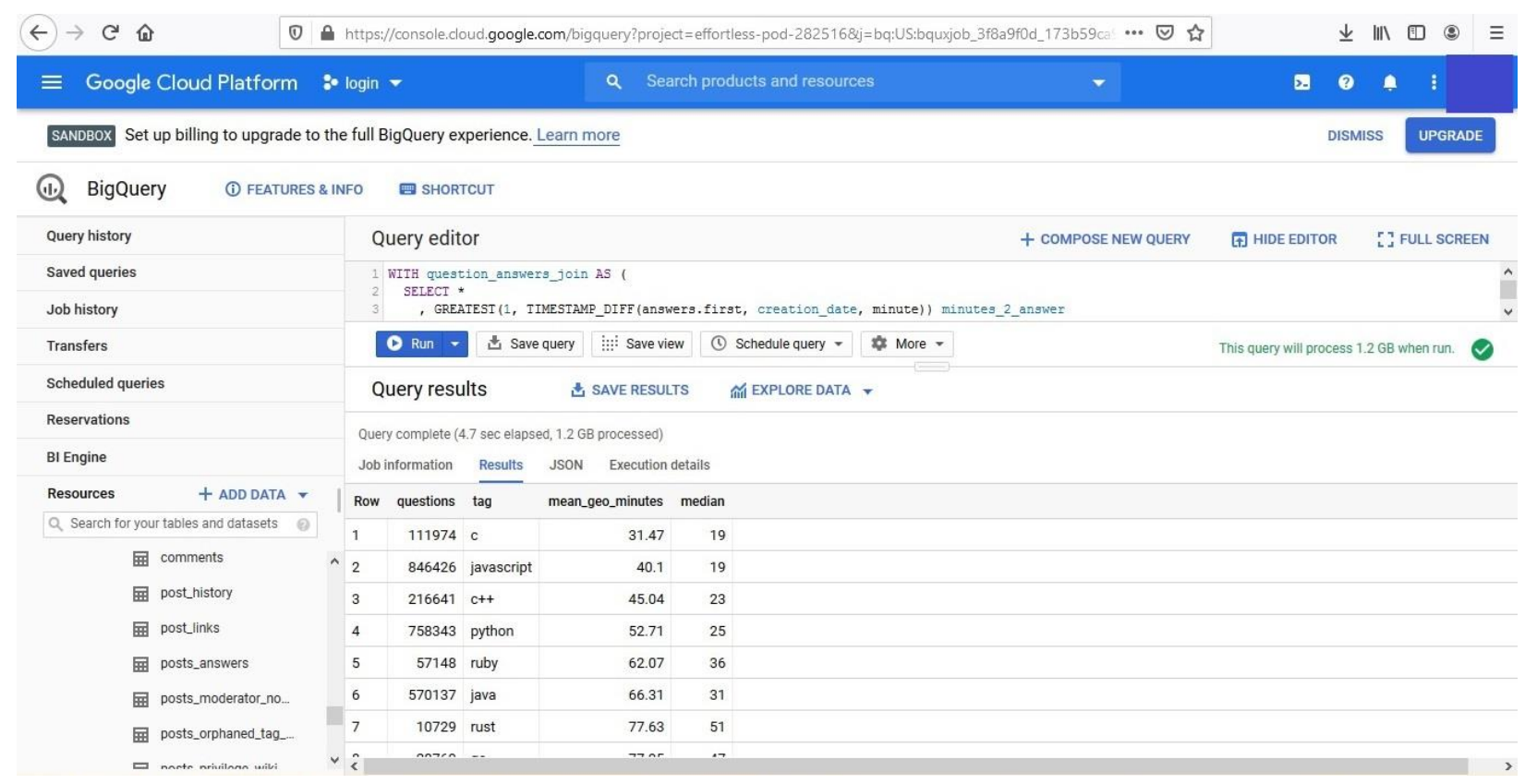

Figure 7 getting result from above fetched query

After clicking the command run the desired output will come in result section refer the above figure. 


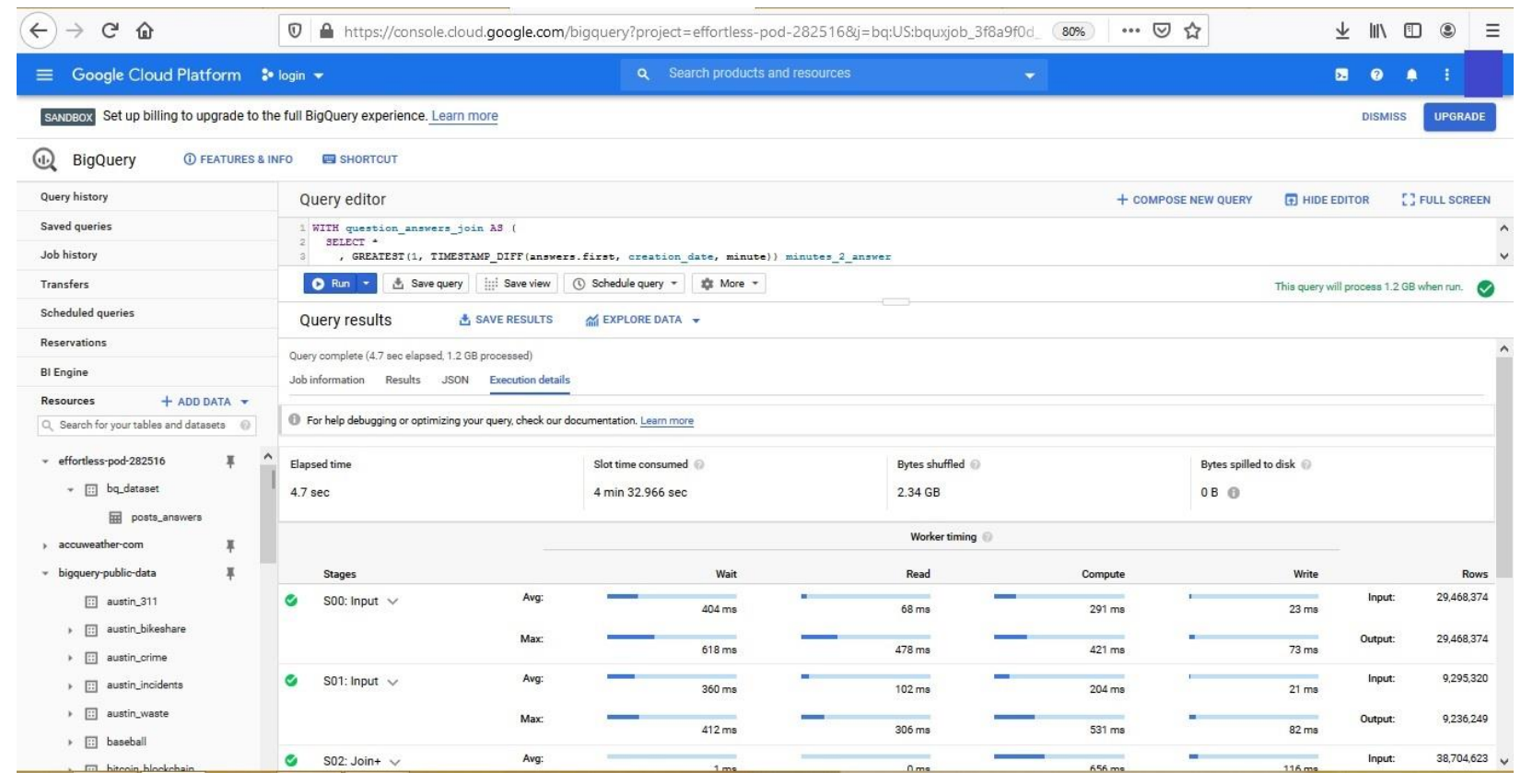

Figure 8 Performance of BigQuery WebUI one

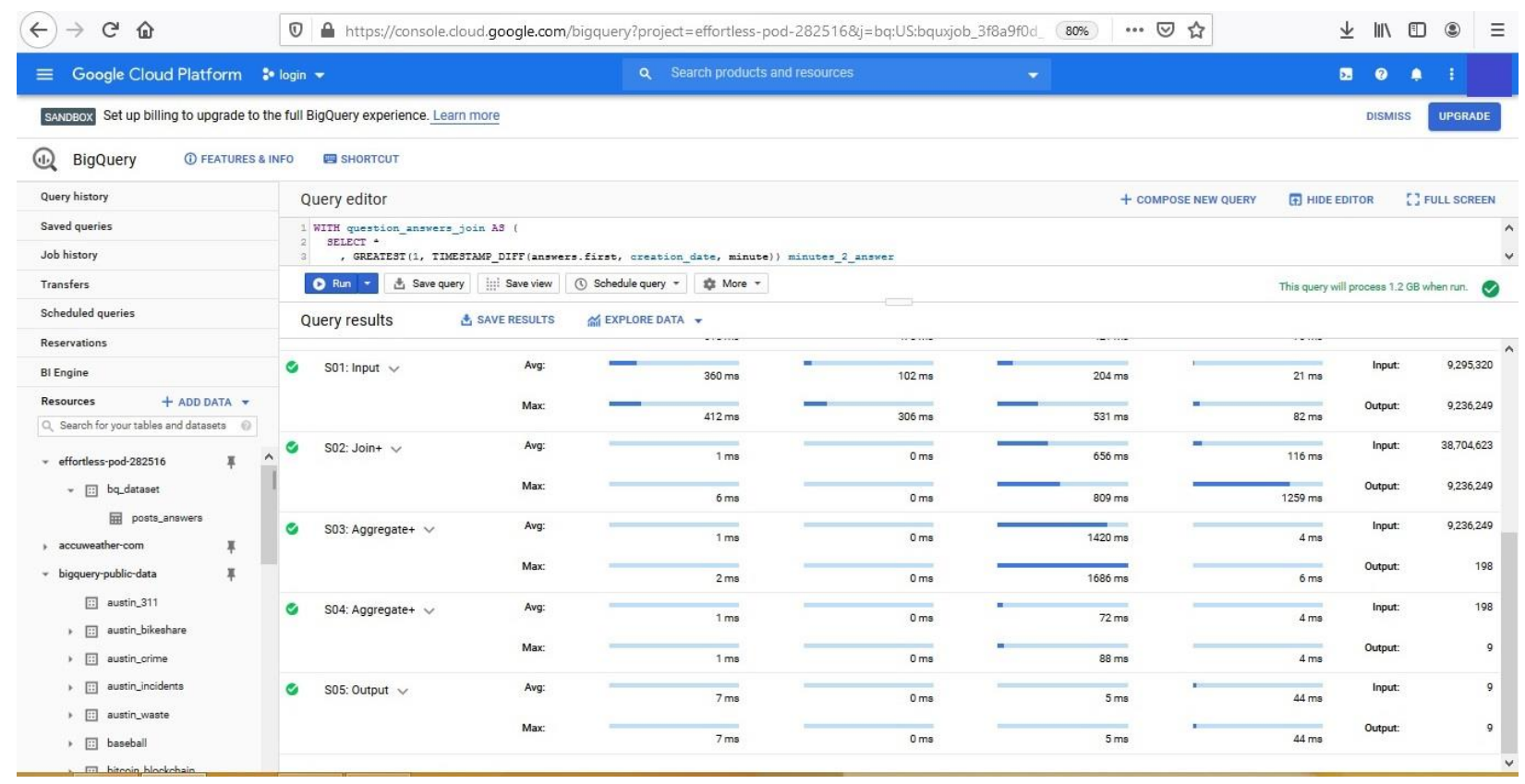

Figure 8 Performance of BigQuery WebUI two

For internal computation of big query how its performing huge amount of data into multiple parallel operations and will fetch all desired output within a friction of seconds. 
Table 2 Query Result

\begin{tabular}{|l|l|l|l|l|}
\hline Row & questions & tag & mean_geo_minutes & median \\
\hline 1 & 111979 & C & 31.42 & 18 \\
\hline 2 & 846423 & JavaScript & 40.12 & 19 \\
\hline 3 & 216641 & C ++ & 45.03 & 22 \\
\hline 4 & 758343 & Python & 52.75 & 24 \\
\hline 5 & 57148 & Ruby & 62.12 & 34 \\
\hline 6 & 570137 & Java & 66.35 & 31 \\
\hline 7 & 10729 & Rust & 77.64 & 53 \\
\hline 8 & 28769 & Go & 77.97 & 44 \\
\hline 9 & 45443 & Scala & 114.42 & 68 \\
\hline
\end{tabular}

Above table is sowing the output of query in to tabular format and below a generated graph based on above table.

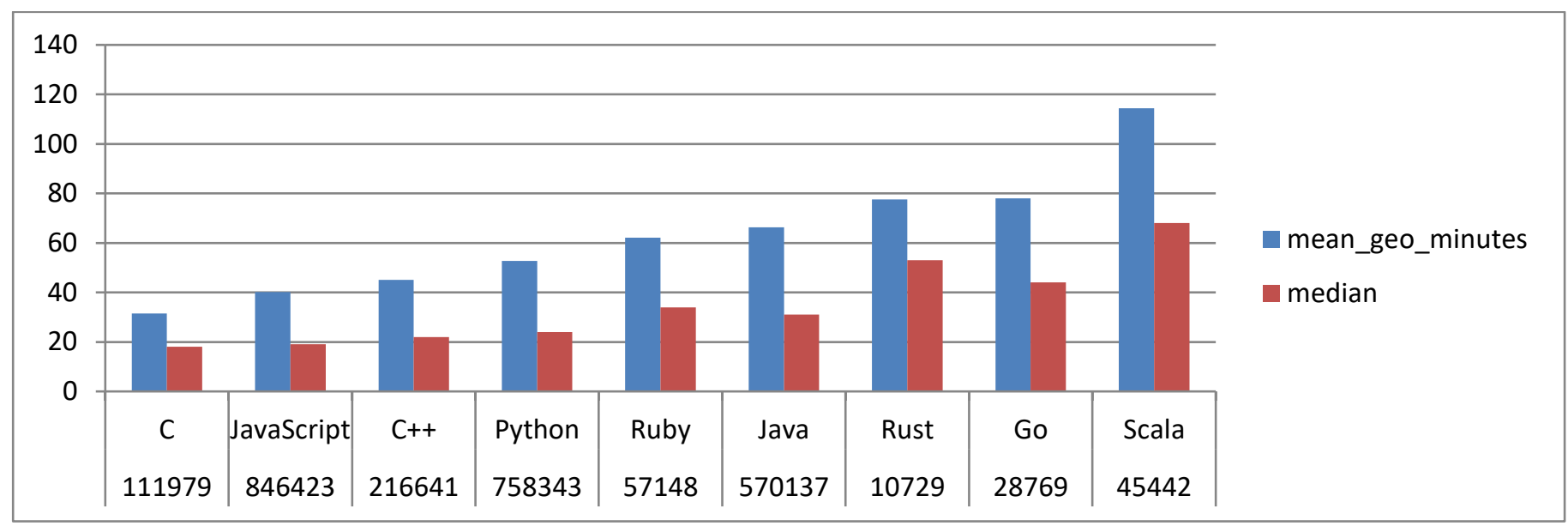

Figure 9 Generated Graph Based on Table-2

\section{Conclusion :}

We have seen by analyze that affecting of request is decreased in each condition, impact will be less on the database where there is even more then 5 stage execution done inside. It have additional consequence on colossal record as stand out from little database. For finishing the inquiry - " discover a language which has the best network, in light of the reaction time", BigQuery took 4.1 seconds and $1.2 \mathrm{~GB}$ information has been examined. After this we can do versatile programming utilizing BigQuery for getting quicker reaction time as contrast with hadoop.

\section{References}

[1]. https://www.sisense.com/blog/thebenefits-of-combining-google-bigquery-andbi/

[2]. Tenzing A SQL Implementation On The MapReduce 
http://research.google.com/pubs/pub37200.ht ml

[3]. Dremel: Interactive Analysis of WebScale Datasets http://research.google.com/pubs/pub36632.ht ml

[4]. Bansari Kotecha, Hetal Joshiyara," Handling Non-Relational Databases on Big Query with Scheduling Approach and Performance Analysis", PG Scholar Computer Science Department,L D College of Engineering, Ahmedabad, India bansari1002@gmail.com,978-1-5386-52572/18/\$31.00 @2018 IEEE

[5]. Nikhil B, Riddhikesh B, Balu P, Mukesh T -A Survey On Scheduling In Hadoop For Bigdata Processing\| Multidisciplinary Journal of Research in Engineering and Technology, Volume 2, Issue 3, pp. 497501,2015, ISSN: 2348 - 6953.

[6]. Yandong Yu, Yuge Yao, "Application of Keyword Dynamic Query Software in Relational Database based on Big Data", Institute of Software, Key Laboratory of Internet of Things at School Level, Jining Normal University Ulanqab, 012000, Inner Mongolia, China, Authorized licensed use limited to: Carleton University. Downloaded on August 11,2020 at 07:05:24 UTC from IEEE Xplore

[7]. S. Yang, Z.G. Chen and N. Xiao, Implementation of directory index for pmfs. Journal of Computer Applications, vol. 37(5), pp. 1241- 1245, 2017.

[8]. E. Dede, M. Govindaraju, D. Gunter, et al., Performance evaluation of a mongodb and hadoop platform for scientific data analysis[C]//Proceedings of the 4th $\mathrm{Acm}$ Workshop on Scientific Cloud Computing. New York:Acm, pp. 13-20, 2013.

[9]. Zheng Youzhuo, Fu Yu, Zhang Ruifeng, Hao Shuqing, Wen Yi, "Research on Lucene Based Full-Text Query Search Service for Smart Distribution

System", Electric Power Research Institute, Guizhou Power Grid Co. Ltd, Guiyang,
China, 2020 3rd International Conference on Artificial Intelligence and Big Data, 978-17281-9741-8/20/\$31.00 C2020 IEEE Authorized licensed use limited to: Macquarie

[10]. S. Lakhara and N. Mishra, "Desktop full-text searching based on Lucene: A review," 2017 IEEE International Conference on Power, Control, Signals and Instrumentation Engineering (ICPCSI), Chennai, 2017, pp. 2434-2438.

[11]. J. Cao, J. Lin, S. Wu, M. Guan, Q. Dai and W. Feng, "Lucene and deep learning based commodity information analysis system," 2016 IEEE International Conference on Consumer Electronics-China (ICCE-China), Guangzhou, 2016, pp. 1-4.

[12]. D. Sloo, N. U. Webb, E. J. Fisher, Y. Matsuoka, A. Fadell, and

M. Rogers, "Smart-home control system providing hvac system dependent responses to hazard detection events," Feb. 27 2018, uS Patent

9,905,122.

[13]. S. Amin and R. Obermaisser, "Timetriggered scheduling of query executions for active diagnosis in distributed real-time systems," in 2017 22nd IEEE International Conference on Emerging Technologies and Factory Automation (ETFA). IEEE, 2017, pp. 1-9.

[14]. Raj ED, L.D DB -A Two Pass Scheduling Policy based Resource allocation for MapReducell International Conference on Information and Communication Technologies (ICICT 2014) Procedia Computer Science, Vol-46 ,pp. 627 634,2015, ISSN:1877 -0509 